\title{
Facial Nerve Reconstruction with Free Vascularized Composite Nerve Flap from Intrapetrous Portion to Terminal Branches-Case Report
}

\author{
Pedro C. Cavadas ${ }^{1,2}$ Magdalena Baklinska ${ }^{1}$ \\ ${ }^{1}$ Clinica Cavadas, Reconstructive Surgery, Valencia, Spain \\ ${ }^{2}$ Departamento de Cirugia, Facultad de Ciencias de la Salud, \\ Universidad Cardenal Herrera-CEU, CEU Universities, Valencia, \\ Spain \\ Indian J Plast Surg 2021;54:204-207.
}

Abstract
Keywords
- facial palsy
- radical parotidectomy
- vascularized nerve
transfer

Address for correspondence Pedro C. Cavadas, MD, PhD, Clinica

Cavadas, Reconstructive Surgery, Valencia 46021, Spain

(e-mail: pcavadas@clinicacavadas.com).
The case presented here is a delayed reconstruction of a facial nerve defect after radical parotidectomy without a useful nerve stump at the stylomastoid foramen. A composite free flap was used to reconnect the nerve's intrapetrous portion to the peripheral branches and reconstruct the soft-tissue deficit.

Facial nerve palsy is a significant disability for most patients, especially young ones. The lack of hemifacial movement may cause social inhibition and a profound psychological disturbance. Impairment in eye protection through deficient blinking mechanism compounds the problem. It is widely accepted that the treatment of facial paralysis depends on the time of denervation. Roughly 1 year is set as the limit after which direct nerve repair should yield to muscle substitution techniques. ${ }^{1,2}$ The injury level also plays a role in the regeneration times, although to a much lesser degree than in longer extremity nerves.

Parotid tumor surgery is one of the leading causes of facial nerve injury, especially en bloc resections. Repair of the resultant nerve defect using nerve grafts (NG) from the main trunk at the stylomastoid foramen to the terminal branches has been widely described with overall good results. ${ }^{3}$ NG depend on the revascularization from the surgical bed and, thus, are not optimal in previously irradiated wound beds. ${ }^{4}$ Taylor and Ham described vascularized nerve transfers (incorrectly named vascularized nerve grafts in the past) first in $1976 .{ }^{5}$ In contrast to NG, the regeneration through vascularized nerve transfers (VNT) is known to be independent of the wound bed's quality. Regenerating axons grow faster through VNT, but the definitive

published online June 30, 2021
DOI https://doi.org/

$10.1055 / \mathrm{s}-0041-1729667$

ISSN 0970-0358 outcome is not significantly better than NG in favorable wound beds. ${ }^{6,7}$ Under certain clinical conditions, a VNT can be superior to NG, that is, in heavily scarred or irradiated areas, in very long defects, when additional tissues are also needed for the reconstruction of composite defects, and probably in cases with long denervation time at presentation, ${ }^{8-10}$ although the level of evidence of this superiority is low.

Reconstruction of extracranial defects of the facial nerve, especially after radical parotidectomy, has been widely described, with some reports on immediate vascularized nerve use with excellent results. ${ }^{11}$ VNT, as part of composite free flaps for simultaneous nerve repair and soft-tissue reconstruction or augmentation, has also been described. ${ }^{12-14}$

The repair of the facial nerve's intracranial injuries, mainly during tumor removal of the posterior fossa with NG from intra- to extracranial portions of the nerve, has been described with good results. ${ }^{15,16}$

The case presented here is a delayed reconstruction of a facial nerve defect after radical parotidectomy without a useful nerve stump at the stylomastoid foramen. A composite free flap was used to reconnect the nerve's intrapetrous portion to the peripheral branches and reconstruct the soft-tissue deficit.
(C) 2021. Association of Plastic Surgeons of India.

This is an open access article published by Thieme under the terms of the Creative Commons Attribution-NonDerivative-NonCommercial-License, permitting copying and reproduction so long as the original work is given appropriate credit. Contents may not be used for commercial purposes, or adapted, remixed, transformed or built upon. (https://creativecommons.org/licenses/by-nc-nd/4.0/).

Thieme Medical and Scientific Publishers Pvt. Ltd. A-12, 2nd Floor, Sector 2, Noida-201301 UP, India 


\section{Case Report}

A 34-year-old woman presented with a right complete facial paralysis after radical parotidectomy and radiation therapy (60 Gy) for parotid adenocarcinoma ( - Fig. 1a, b). The denervation time was 13 months. The parotid region was depressed and heavily scarred, and according to the previous operative notes, the facial nerve had been resected flush to the stylomastoid foramen. No perineural infiltration was present in the pathology report. No additional electrophysiologic tests were deemed necessary for decision-making in this case.

Given the long denervation time, the irradiated area, and the unlikely presence of a suitable proximal extracranial nerve stump, a vascularized nerve reconstruction was planned. Though the previous parotidectomy scar, with a postauricular extension, the distal five main branches of the facial nerve (frontal, orbital, zygomatic, buccal, and marginal mandibular) were identified and tagged. A standard mastoidectomy was performed to expose the third portion of the intrapetrous facial nerve. The nerve was sectioned in the midpoint of this portion and examined visually at high magnification to confirm a healthy fascicular pattern. Using the right lateral circumflex femoral vessels (LCFV), a composite flap was harvested consisting of two adipofascial islands (one based on the anterolateral tight [ALT] perforator and the other on a branch to the tensor fasciae lata), and a $13-\mathrm{cm}$ segment of the motor nerve of the vastus lateralis $(\mathrm{VL})$ was dissected distally to include five distal branches with their associated vascular supply (-Fig. 2). The right lingual artery and vein were dissected as recipients.

The proximal end of the VL motor nerve was coapted to the intrapetrous facial nerve with epineural $10 / 0$ nylon sutures under the microscope ( $\mathbf{- \text { Fig. }} \mathbf{3}$ ). The distal five branches of the VL nerve were coapted to the facial nerve's distal five branches with 10/0 nylon. The flap's soft-tissue islands were used for filling the resultant dead space after the mastoidectomy and for subcutaneous augmentation of the parotid area ( - Fig. 4). The flap vessels were anastomosed end-to-end to the lingual artery and vein. A portion of fat tissue was left exposed in the mastoid region for monitoring and left to heal secondarily. The postoperative course was uneventful. Free ambulation was allowed at 2 weeks, and unrestricted physical activity at 1 month.

At four months follow-up, the symmetry of the face was recovered at rest ( $\mathbf{- F i g}$. 5). The voluntary function of the lower four branches improved steadily afterward. The frontal
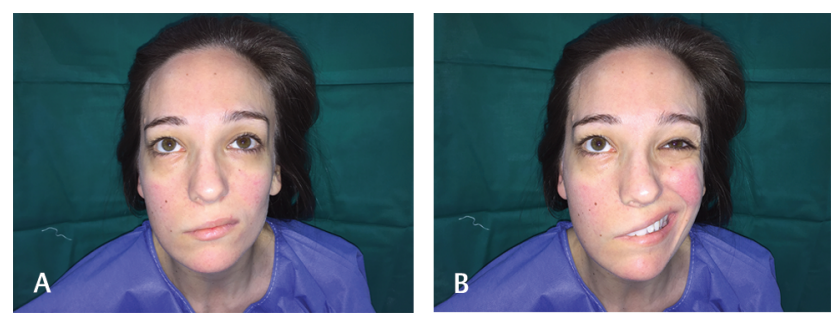

Fig. 1(A,B) Preoperative image of the patient with 13-month-old right facial paralysis due to radical parotidectomy and radiation therapy.

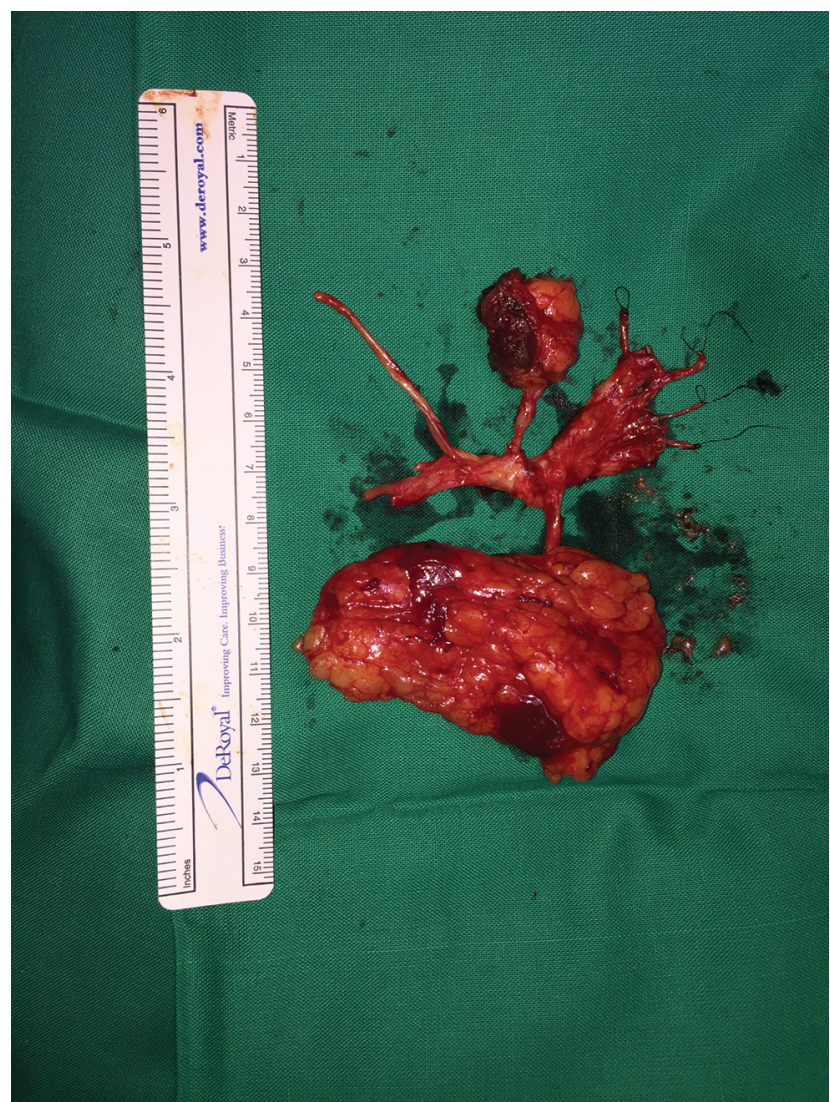

Fig. 2 A composite free flap from the lateral circumflex femoral vessels, including two soft-tissue islands and a $13-\mathrm{cm}$-long segment of the motor nerve of the vastus lateralis with five distal branches, was elevated from the right side.

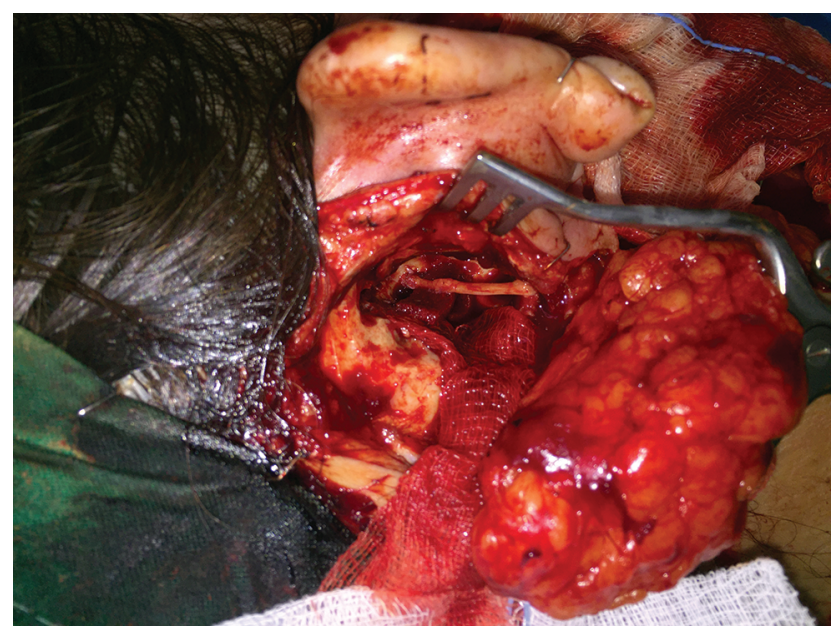

Fig. 3 After radical mastoidectomy and drilling of the third portion of the intrapetrous facial nerve, the proximal nerve repair was performed with $10 / 0$ nylon sutures.

branch did not recover function. At 3 years of follow-up, active facial nerve function was grade II in the House-Brachmann score ${ }^{17}$ in the lower branches but no function in the frontal branch. An eyebrow pexy was performed at 2 years to palliate the eyebrow asymmetry ( - Fig. 6 ). No identifiable functional donor deficit was reported by the patient. 


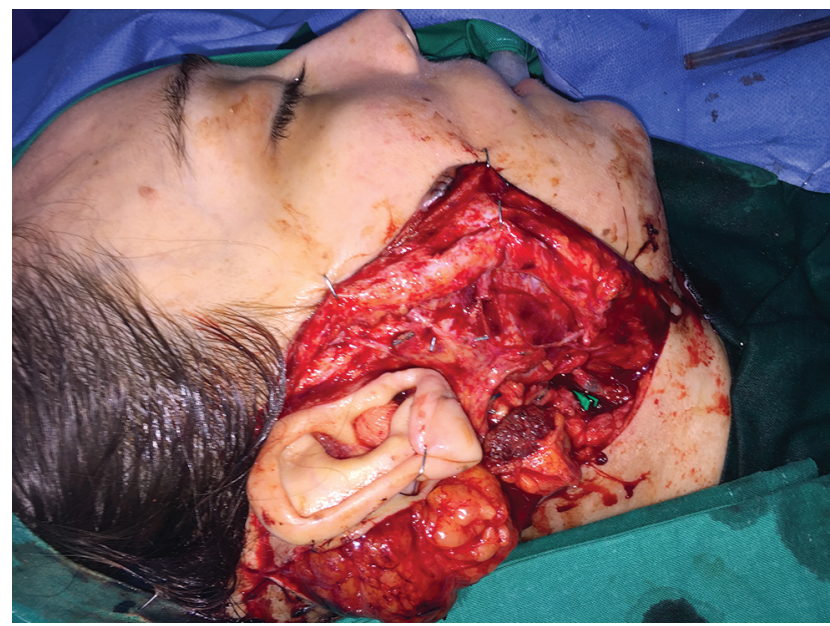

Fig. 4 The distal branches of the motor nerve of the vastus lateralis were coapted to the five distal main branches of the facial nerve with $10 / 0$ nylon. The mastoidectomy defect and the parotid area were reconstructed with the two soft-tissue islands of the flap. The flap was revascularized to the lingual vessels.
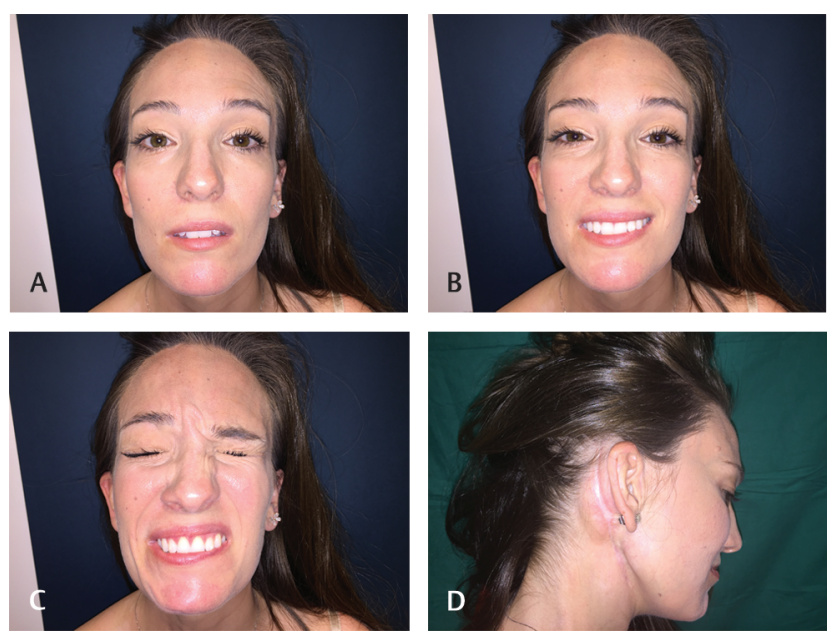

Fig. 6 Function at 3 years at rest (a), mild contraction (b), and forced contraction (c). The profile of the mastoid defect and the parotid area was acceptable to the patient $(\mathbf{d})$.

\section{Discussion}

Facial nerve palsy in a young patient can be devastating from a social and psychological standpoint. Multiple treatment options exist, depending basically on the denervation time, the limit for nerve repair being roughly 1 year. ${ }^{1,2}$ Late (over 12 months) paralysis has a worse prognosis since reinnervation is less predictable, and functioning muscle transfers have inferior results compared with early nerve repair. ${ }^{2}$ The case presented was past the upper limit for direct nerve reconstruction, but given the patient's age and gender, the best possible result was aimed. Time was a primary concern in this case, and the reported faster axonal growth in VNT compared with NG was considered in the planning. ${ }^{6.7}$ The irradiated field and the concomitant need for soft-tissue augmentation in the parotid region also favored a VNT as part of the composite free flap. ${ }^{12-14}$

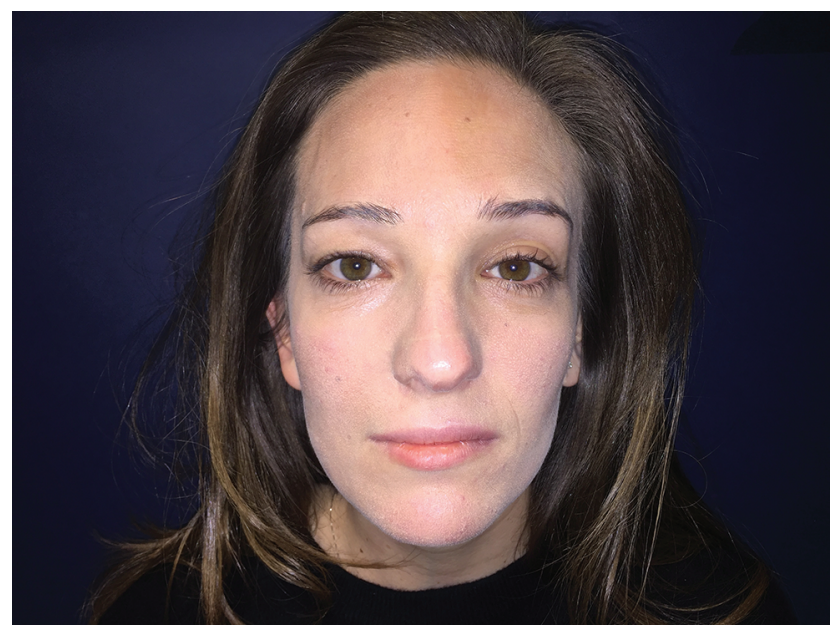

Fig. 5 At 4 months, the symmetry of the face at rest was recovered.

Reconstruction of nerve defects after oncological resection of parotid gland malignancies usually entails nerve repair from the facial trunk near the stylomastoid foramen to the five main distal branches. NG and VNT have been described in this setting with overall good results, especially in immediate reconstructions. ${ }^{11}$ When the proximal stump cannot be identified (usually in delayed cases), cross-facial grafts, or crossover transfers (masseteric nerve) are the usual treatments. ${ }^{2}$ Intra-extra cranial NG have been described for acute repair of intradural or intrapetrosal injuries with acceptable results. ${ }^{15,16}$ Because these are immediate reconstructions, the time of regeneration is less of a concern.

There is a growing body of evidence of the noninferiority of expendable motor nerves compared with the more popular sensory nerves as donors for NG. The avoidance of skin hypoesthesia and possible painful neuroma and limited donor morbidity could be advantageous. Thoracodorsal nerve or portions of the femoral nerve have been investigated as donor areas for NG. ${ }^{18-20}$ Motor nerves as VNT have also been described either alone or as a part of composite free tissue transfers for facial defects. ${ }^{21,22}$

When the extracranial portion of the facial nerve is not usable, using the intrapetrous portion through mastoidectomy and petrous drilling seems logical to have a native ipsilateral proximal nerve stump, even at the expense of increasing the length of the nerve defect. In this case, the healthy intrapetrous facial nerve was the optimal choice for direct nerve reconstruction. Training in skull base approaches and familiarity with mastoidectomy and facial nerve drilling is advantageous for reconstructive surgeons. Otherwise, ENT surgeons should be consulted. Other alternatives of treatment as cross facial NG would have taken too long to regenerate in this case and would have likely resulted in poor function. The possibility of recycling these grafts as recipients for an eventual free functional muscle transfer in case of failure to reinnervate could be part of this hypothetical alternative. Closer-target nerve transfers, that is, masseteric nerve, would take shorter to grow into the ipsilateral facial nerve, 
but in this case, NG would have been necessary to reach the five distal branches since the main facial trunk was missing. This option was, therefore, discarded. The increase in the nerve defect's length using the intrapetrous facial nerve can be compensated for by the faster axonal growth in VNT and still achieve a native facial nerve restoration. To the best of the authors' knowledge, a VNT from the intrapetrous facial nerve to terminal branches has not been described before.

\section{Conflict of Interest}

None declared.

\section{References}

1 Gao Z, Jia XH, Xu J, et al. Neurorrhaphy for facial reanimation with interpositional graft: outcome in 23 patients and the impact of timing on the outcome. World Neurosurg 2019;126:e688-e693

2 Hadlock TA, Cheney ML. Update on facial nerve repair. Facial Plast Surg 1998;14(3):179-184

3 Ch'ng S, Ashford BG, Gao K, McGuinness J, Clark JR. Reconstruction of post-radical parotidectomy defects. Plast Reconstr Surg 2012;129(2):275e-287e

4 Prpa B, Huddleston PM, An KN, Wood MB. Revascularization of nerve grafts: a qualitative and quantitative study of the soft-tissue bed contributions to blood flow in canine nerve grafts. J Hand Surg Am 2002;27(6):1041-1047

5 Taylor GI, Ham FJ. The free vascularized nerve graft. A further experimental and clinical application of microvascular techniques. Plast Reconstr Surg 1976;57(4):413-426

6 Schultes G, Gaggl A, Kleinert R, Karcher H. Vascularized versus non-vascularized nerve transfers: histologic study in rats. J Reconstr Microsurg 2001;17(8):637-642

7 Koshima I, Harii K. Experimental study of vascularized nerve grafts: morphometric study of axonal regeneration of nerves transplanted into silicone tubes. Ann Plast Surg 1985;14(3):235-243

8 Mackinnon SE, Kelly L, Hunter DA. Comparison of regeneration across a vascularized versus conventional nerve graft: case report. Microsurgery 1988;9(4):226-234

9 Doi K, Tamaru K, Sakai K, Kuwata N, Kurafuji Y, Kawai S. A comparison of vascularized and conventional sural nerve grafts. J Hand Surg Am 1992;17(4):670-676

10 Koshima I, Nanba Y, Tsutsui T, Takahashi Y, Kawai A. Vascularized femoral nerve graft with anterolateral thigh true perforator flap for massive defects after cancer ablation in the upper arm. J Reconstr Microsurg 2003;19(5):299-302

11 Kimata Y, Sakuraba M, Hishinuma S, Ebihara S, Hayashi R, Asakage T. Free vascularized nerve grafting for immediate facial nerve reconstruction. Laryngoscope 2005;115(2):331-336

12 Iida T, Nakagawa M, Asano T, Fukushima C, Tachi K. Free vascularized lateral femoral cutaneous nerve graft with anterolateral thigh flap for reconstruction of facial nerve defects. J Reconstr Microsurg 2006;22(5):343-348

13 Hyodo I, Ozawa T, Hasegawa Y, Ogawa T, Terada A, Torii S. Management of a total parotidectomy defect with a gastrocnemius muscle transfer and vascularized sural nerve grafting. Ann Plast Surg 2007;58(6):677-682

14 Kashiwa K, Kobayashi S, Nasu W, Kuroda T, Higuchi H. Facial nerve reconstruction using a vascularized lateral femoral cutaneous nerve graft based on the superficial circumflex iliac artery system: an application of the inferolateral extension of the groin flap. J Reconstr Microsurg 2010;26(9):577-582

15 Samii M, Matthies C. Management of 1000 vestibular schwannomas (acoustic neuromas): the facial nerve-preservation and restitution of function. Neurosurgery 1997;40(4):684-694, discussion 694-695

16 Prasad SC, Balasubramanian K, Piccirillo E, et al. Surgical technique and results of cable graft interpositioning of the facial nerve in lateral skull base surgeries: experience with 213 consecutive cases. J Neurosurg 2018;128(2):631-638

17 House JW. Facial nerve grading systems. Laryngoscope 1983;93(8):1056-1069

18 Nichols CM, Brenner MJ, Fox IK, et al. Effects of motor versus sensory nerve grafts on peripheral nerve regeneration. Exp Neurol 2004;190(2):347-355

19 Ali SA, Rosko AJ, Hanks JE, et al. Effect of motor versus sensory nerve autografts on regeneration and functional outcomes of rat facial nerve reconstruction. Sci Rep 2019;9(1):8353

20 Biglioli F, Colombo V, Pedrazzoli M, et al. Thoracodorsal nerve graft for reconstruction of facial nerve branching. J Craniomaxillofac Surg 2014;42(1):e8-e14

21 Bedarida V, Qassemyar Q, Temam S, Janot F, Kolb F. Facial functional outcomes analysis after reconstruction by vascularized thoracodorsal nerve free flap following radical parotidectomy with facial nerve sacrifice. Head Neck 2020;42(5):994-1003

22 Xu ZF, Duan WY, Tan XX, Sun CF. Reconstruction of complex total parotidectomy defect with a chimeric anterolateral thigh perforator flap and vascularized motor branch of femoral nerve grafting. J Oral Maxillofac Surg 2015;73(12):2448. e1-2448.e7 